\title{
Solution to Problem 11275
}

David H. Bailey ${ }^{1}$ and Jonathan M. Borwein ${ }^{2}$

Problem 11275, which appears in the February 2007 issue of the American Mathematical Monthly, asks to evaluate the iterated integral

$$
G:=\int_{0}^{\infty} \int_{y}^{\infty} \frac{(x-y)^{2} \log ((x+y) /(x-y))}{x y \sinh (x+y)} d x d y
$$

Answer. When this issue of the Monthly arrived in the mail, both of the present authors recognized that this problem was amenable to experimental methods, and independently began to work on it. Bailey set out to calculate the original double integral, after making the minor substitution $u=x-y$, so that both integrals have constant limits. This effort produced the numerical result

$$
G=1.1532659890804730178602752931059938854511244009224435425100 \ldots
$$

Bailey tried using the Inverse Symbolic Calculator (ISC), an online numeric constant recognition tool available at http://oldweb.cecm.sfu.ca/projects/ISC/ISCmain.html, but it was not able to recognize this constant.

Meanwhile, Borwein, working in Maple, employed the simple substitution $x=t y$ to write

$$
G=\int_{0}^{\infty} \int_{1}^{\infty} \frac{y(t-1)^{2} \log ((t+1) /(t-1))}{t \sinh (t y+y)} d t d y
$$

He then interchanged the order of integrals to produce the 1-D integral

$$
G=\frac{\pi^{2}}{4} \int_{1}^{\infty} \frac{(t-1)^{2}(\log (t+1)-\log (t-1))}{t(t+1)^{2}} d t
$$

which after the substitution $t=1 / s$ yielded

$$
G=\frac{\pi^{2}}{4} \int_{0}^{1} \frac{(s-1)^{2}(\log (1+s)-\log (1-s))}{s\left(1+s^{2}\right)} d s .
$$

Now Maple was able to numerically evaluate either form of the single integral integral (without the external coefficient) as $0.4674011002723397 \ldots$, and further was able to recognize this constant as $\pi^{2} / 4-2$, via the identify function (Mathematica has a Recognize operator, but it can only find algebraic numbers).

Thus, the entire integral was recognized as

$$
G=\frac{\pi^{4}}{16}-\frac{\pi^{2}}{2}
$$

\footnotetext{
${ }^{1}$ Lawrence Berkeley National Laboratory, Berkeley, CA 94720. dhbailey@lbl.gov. This work was supported by the Director, Office of Computational and Technology Research, Division of Mathematical, Information, and Computational Sciences of the U.S. Department of Energy, under contract number DE-AC02$05 \mathrm{CH} 11231$.

${ }^{2}$ Faculty of Computer Science, Dalhousie University, Halifax, NS, B3H 2W5, Canada. jborwein@cs.dal.ca. This work supported in part by NSERC and the Canada Research Chair Programme.
} 
Indeed, with more care and hindsight, the single Maple instruction identify(1.15326598908047301786027, BasisSizePoly=7); immediately returns (1).

Now that Borwein "knew" the answer, it was a fairly simple matter, still working in a Maple environment, to "prove" it. This was done by substituting $u=(1-s) /(1+s)$ in the third form above to yield the simple equivalent form

$$
G=\frac{\pi^{2}}{2} \int_{0}^{1} \frac{u^{2} \log u}{u^{2}-1} d u
$$

which Maple was able to evaluate analytically to produce the closed-form result, and which one can do by hand - say by using the geometric series and integrating term-by-term: the human proof of the last step is

$$
\begin{aligned}
\int_{0}^{1} \frac{u^{2} \log u}{u^{2}-1} d u & =-\sum_{n=1}^{\infty} \int_{0}^{1} u^{2 n} \log u d u \\
& =\sum_{n=1}^{\infty} \frac{1}{(2 n+1)^{2}}=\frac{\pi^{2}}{8}-1 .
\end{aligned}
$$

Conclusion. Two-dimensional numerical quadrature and one line of Maple code provide the evaluation but no proof. Maple with limited hand coaxing provides a proof which can then either be automated or made human. 